Revista Electrónica Complutense de Investigación en Educación Musical ISSN-e: 1698-7454

http://dx.doi.org/10.5209/RECIEM.52409

\title{
Documentos clave de la Unión Europea sobre educación musical en las enseñanzas obligatorias
}

\author{
Narciso José López García; ${ }^{1}$ María del Valle De Moya Martínez
}

Recibido: 30 de abril de 2016 / Aceptado: 30 de noviembre de 2016

Resumen: La Unión Europea, asumiendo las directrices de la UNESCO sobre la importancia de la Educación Artística en el desarrollo integral de la persona, lleva décadas trabajando por la plena inclusión de esta área de conocimiento en los currículos de sus estados miembros y encargando a sus instituciones educativas una serie de informes que recojan el estado actual de esta materia en los sistemas educativos de los veintiocho, con la idea de conocer su configuración en las normas educativas de los mismos para, posteriormente y tras su estudio, lanzar las propuestas de mejora oportunas que permitan consolidar su presencia en los currículos nacionales. Nuestro trabajo se ha basado en recoger y analizar los aspectos más significativos publicados en estos textos y en seleccionar aquellos que, por su contenido específico, mejor reflejan la postura comunitaria sobre la educación musical en las enseñanzas básicas, ofreciendo una breve reseña de los once documentos que ofrecen, a nuestro parecer, los aspectos más relevantes sobre las políticas educativo-musicales que se vienen desarrollando en el ámbito comunitario, cómo estos han influido en los diseños curriculares nacionales y las conclusiones generales derivadas de este proceso analítico.

Palabras clave: Educación musical, Unión Europea, sistemas educativos, política educativa, toma de decisiones.

\section{[en] Key documents from European Union about Music Education in compulsory education}

\begin{abstract}
The European Union, assuming UNESCO guidelines on the importance of Art Education in children's integral development, has spent decades working for the full inclusion of this area of knowledge in the curricula of its member States and asking their educational institutions for a series of reports containing the current status of this matter in the educational systems of the twenty-eight, in order to know its configuration in their educational rules, and to launch appropriate proposals for improvement that will allow to consolidate its presence in the national curricula. Our work is based on collecting and analyzing most significant aspects published in these texts and selecting those which, due to their specific content, reflect the EU position on Music Education in basic teachings, providing a brief overview of the eleven documents they offer, in our view, the most relevant aspects of educational music policies that are being developed on the EU scale, how these ones have influenced national curricula and general conclusions derived from this analytical process.
\end{abstract}

Keywords: Music education, European Union, educational systems, educational policy, decision making.

\footnotetext{
1 Facultad de Educación de Albacete (Universidad de Castilla-La Mancha).

E-mail: narcisojose.lopez@uclm.es

2 Facultad de Educación de Albacete (Universidad de Castilla-La Mancha).

E-mail: mariavallede.moya@uclm.es
} 
Sumario: 1. Introducción. 2. Origen y evolución de la unión europea: breve reseña histórica. 3. Breve acercamiento a la política educativa de la Unión Europea. 4. Estrategia metodológica y fuentes de información. 5. Once documentos clave de la Unión Europea sobre educación musical. 6. Conclusiones. 7. Limitaciones y prospectivas. 8. Referencias bibliográficas.

Cómo citar: López García, N.J. y De Moya Martínez, M.V. (2017). Documentos clave de la Unión Europea sobre educación musical en las enseñanzas obligatorias. RECIEM. Revista Electrónica Complutense de Investigación y Educación Musical, 14, 171-186.

\section{Introducción}

El Congreso Internacional sobre el Papel y el Lugar de la Música en la Educación de Jóvenes y Adultos, convocado por la UNESCO en 1953, significó un punto de inflexión que marcaba un antes y un después en la consideración curricular de la Educación Musical en los procesos de enseñanza-aprendizaje y en la necesidad de su presencia en los sistemas educativos.

Desde entonces hasta nuestros días, tanto la UNESCO como otras instituciones de reconocimiento internacional, vienen desarrollando diferentes acciones enfocadas a concienciar a los distintos gobiernos de la importancia de impulsar políticas educativas comprometidas con la implantación, en unos casos, y con la mejora, en otros, de esta disciplina de conocimiento en los currículos como parte fundamental del desarrollo integral de la persona. En este sentido, la Unión Europea, comprometida con la UNESCO, recoge y asume las distintas recomendaciones que esta organización propone en materia educativa, y mantiene una preocupación constante por que sus estados miembros adapten e incluyan estas consideraciones en sus sistemas educativos nacionales.

Fruto de este empeño, han surgido numerosos informes que, tanto la Red Eurydice como el resto de instituciones y organismos de la Unión especializados en política educativa, vienen publicando sobre aquellos temas relacionados con la educación que se consideran de alto interés comunitario. Algunos de estos documentos hacen referencia a la educación musical y reflejan la postura de la UE sobre la importancia de esta materia en los procesos de enseñanza-aprendizaje, especialmente en las etapas educativas que comprenden al alumnado de edades más tempranas.

Si bien, presentar todos estos escritos se aleja, por razones obvias, de nuestro objetivo, hemos seleccionado once informes relacionados con la enseñanza de la música que, desde nuestro punto de vista, mejor definen la posición de la Unión en este asunto. Pero antes, hemos creído conveniente acercarnos brevemente a la realidad de esta institución y a su papel en materia educativa, lo que nos ayudará a entender algunas de las controvertidas decisiones que se han tomado en los últimos meses en algunos estados miembros sobre la presencia curricular de la Educación Musical en las enseñanzas de régimen general no universitarias, que se han intentado justificar en virtud de las competencias exclusivas que estos países siguen manteniendo. 


\section{Origen y evolución de la Unión Europea: breve reseña histórica}

Tras la II Guerra Mundial, Jean Monet (considerado el padre de la Unión Europea) y Robert Schuman, ministro francés de Asuntos Exteriores, propondrán una vía para la construcción de una nueva Europa basada en realizaciones limitadas pero concretas y siempre desde una perspectiva de solidaridad entre estados. Esta propuesta quedará plasmada en la Declaración Schuman de 1950, que dará lugar a la firma del Tratado de París de 1951, en el que Francia, Alemania, Italia, Países Bajos, Bélgica y Luxemburgo se comprometen a organizar, de manera conjunta, la libre circulación del carbón y del acero y al libre acceso a sus fuentes de producción.

A partir de este momento, y hasta 1992, se aprobarán varios tratados y se producirán nuevas adhesiones, dando lugar a un naciente panorama europeo que abrirá las puertas a nuevas acciones sobre creación de políticas consensuadas, que quedarán plasmadas en el Tratado de Maastricht o Tratado de la Unión Europea, el cual apuesta por una auténtica unión de países en la que los factores políticos tienen cada vez más peso y cuya estructura interna se basa en tres pilares fundamentales:

- El ámbito comunitario basado en el principio de cohesión económica y social.

- El ámbito de política exterior y de seguridad común.

- El ámbito de cooperación en asuntos de justicia e interior.

Estos tres ámbitos darán solidez al nuevo concepto de ciudadanía europea, basado en el respeto a los derechos y libertades fundamentales y en el respeto a la identidad nacional de sus miembros.

Entre los años 1995 y 2013 se han ido produciendo nuevas adhesiones hasta alcanzar un total de veintiocho estados y, actualmente, "la Unión Europea está abierta a todo país europeo que cumpla los criterios democráticos, políticos y económicos de adhesión" (Fontaine, 2011, p. 14).

Mención especial merece el Proceso de Lisboa de 2000, que marcaría las estrategias a seguir con la mirada puesta en el año 2020. Estas estrategias tienen por objeto desarrollar en estos veinte años, por un lado, actuaciones que respondan a la globalización y a la crisis económica haciendo de nuevo competitiva a la economía europea, y por otro lado, intervenciones que garanticen el crecimiento inteligente, sostenible e inclusivo. Para alcanzar estos objetivos, el Proceso de Lisboa "abarcaba acciones en una amplia serie de ámbitos, como la educación, la formación profesional, la investigación científica, el acceso a Internet y las transacciones en línea, e incluía igualmente la reforma de los sistemas de seguridad social europeos" (Fontaine, 2011, p. 49).

Las nuevas circunstancias que se han ido presentando en estos primeros años del siglo XXI están obligando a las instituciones de la Unión a proponerse nuevos retos, nuevas posibilidades, que le permitan llevar a término la misión que se ha propuesto en este siglo y que se pueden resumir en estos seis grandes bloques:

- Mantener y aprovechar la paz establecida entre sus estados miembros.

- Unir a los países europeos en una cooperación práctica.

- Velar por que los ciudadanos europeos puedan vivir con seguridad.

- Promover la solidaridad económica y social.

- Preservar la identidad y la diversidad europeas en un mundo globalizado.

- Promulgar los valores compartidos por los europeos. 
La progresiva consecución de estos seis aspectos tiene como objetivo fundamental crear el sentimiento de identidad común, imprescindible para la consolidación de la gobernanza económica, para un crecimiento más fuerte y para una mayor atención a los ciudadanos de la Unión Europea como medidas que pueden aliviar sus preocupaciones cotidianas (Comisión Europea, 2012).

\section{Breve acercamiento a la política educativa de la Unión Europea}

El artículo 6 de la Versión consolidada del Tratado de Funcionamiento de la Unión Europea (TFUE, 2010) señala que:

La Unión dispondrá de competencia para llevar a cabo acciones con el fin de apoyar, coordinar o complementar la acción de los estados miembros. Los ámbitos de estas acciones serán, en su finalidad europea: [...] e) La educación, la formación profesional, la juventud y el deporte.

Expertos como Diestro (2009), Etxeberria, Ayerbe, Garagorri y Vega (2000) y Paricio (2005), entre otros, coinciden en que esta exposición de intenciones y modos de actuación especifica claramente que la política educativa de los estados miembros es de competencia nacional, aunque pone de manifiesto la facultad y el compromiso de la Unión de complementarla a través de acciones y programas concretos.

Desde esta perspectiva, la estructura, administración, financiación, organización y currículo de los sistemas educativos nacionales son, exclusivamente, potestad de los estados miembros, por lo que la política educativa de la Unión Europea hay que entenderla como una serie de orientaciones cuya pretensión no va más allá de ser un mero apoyo y complemento, siempre desde los principios de subsidiariedad, proporcionalidad y respeto absoluto a las competencias nacionales, a las acciones de los estados miembros para que éstas guarden una mayor coherencia entre sí (Valle, 2004). Estos principios no le permiten legislar, pero sí velar por que las legislaciones educativas de los veintiocho favorezcan la creación de un espacio europeo único, siendo éste el objetivo último de la política educativa de la Unión.

En este sentido, sus organismos e instituciones educativas trabajan por una educación de calidad mediante el desarrollo de diferentes programas que se encargan de reforzar los intercambios educativos, fomentar la financiación a proyectos de cooperación, de la enseñanza de idiomas, del aprendizaje electrónico, del intercambio de prácticas y de dar consistencia al sentimiento de identidad europea.

Tabla 1: Organismos e Instituciones de la UE con competencias en política educativa

\begin{tabular}{|l|l|}
\hline Organismos e Instituciones & Comisiones encargadas \\
\hline Comisión Europea & Comisión de Educación y Formación \\
\hline Parlamento Europeo & Comisión de Cultura y Educación \\
\hline Consejo de la UE & Comisión de Educación, Juventud y Cultura \\
\hline Comité de las Regiones & Comisión de Educación, Juventud, Cultura e Investigación \\
\hline Banco Europeo de Inversiones & Iniciativa de Investigación BE de Inversiones - Universidades \\
\hline Agencias de la UE & $\begin{array}{l}\text { CEDEFOP, EACEA, Fundación Europea de Formación, Eury- } \\
\text { dice, EIT }\end{array}$ \\
\hline
\end{tabular}


A su vez, y desde la firma del Tratado Constitutivo de la Comunidad Económica Europea (CEE) de 1957 -en el que aparecen unas escuetas referencias sobre educación- la Unión Europea ha emitido (y lo sigue haciendo), miles de documentos relacionados con su política en materia de educación, cumpliendo así con su función de apoyar y complementar el trabajo de los estados miembros en este ámbito (Valle, 2006a). De todos ellos, son unos treinta los que, con mayor o menor profundidad, desarrollan la postura europea sobre la importancia de la enseñanza de la música en la educación obligatoria, de los que hemos seleccionado once textos imprescindibles que los ministerios de educación nacionales deberían tener en cuenta en futuras modificaciones curriculares.

\section{Estrategia metodológica y fuentes de información}

Nuestro trabajo se ha llevado a cabo desde un enfoque cualitativo basado en el análisis de información, entendido éste como la forma de investigar cuyo objetivo consiste en captar, evaluar, seleccionar y sintetizar los mensajes contenidos en una serie de documentos (Dulzaides y Molina, 2004). Esta estrategia metodológica está emparentada con los análisis de contenido y documental, en los que la descripción y la interpretación de la información y de las ideas contenidas en los textos a estudio se han configurado como eje principal de nuestra investigación desde un enfoque sistemático y objetivo (Bardin, 2002).

El escaso número de trabajos, investigaciones y estudios actualizados relacionados con el tema que nos ocupa, nos ha obligado a realizar una recogida de información mediante la exploración exhaustiva de documentos y legislación que proporcionan las diferentes instituciones europeas con competencias en materia educativa, así como mediante la búsqueda específica de textos relacionados con la educación musical a través de los buscadores web que ofrecen dichas instituciones. Además, algunos de los textos con los que hemos trabajado se han recogido de la red Eurydice, organismo que, desde 1980, proporciona información actualizada sobre los sistemas y políticas educativas de treinta y siete países europeos, en los que están incluidos los veintiocho estados miembros. Esta red tiene dos objetivos fundamentales:

- Proporcionar a los responsables de los sistemas educativos europeos información que ayude en la toma de decisiones a nivel legislativo.

- Proporcionar a cualquier ciudadano, pertenezca o no a la Unión, información sobre los sistemas educativos de los treinta y siete países que participan en este proyecto, ofreciendo descripciones detalladas de los sistemas nacionales, informes temáticos y comparativos sobre temas específicos relacionados con la educación que son de interés comunitario y datos generales relacionados con los calendarios escolares, comparación de horas lectivas o niveles educativos, entre otros datos.

Por otro lado, hemos querido conocer el estado actual de la Educación Musical en la enseñanza primaria en los currículos oficiales de los veintiocho, dato fundamental para entender el grado de significación de los documentos seleccio- 
nados en la realidad educativo-musical de la Unión. Para ello, se han analizado los programas ofrecidos por los diferentes ministerios nacionales en sus webs. A su vez, enviamos a los mismos un sencillo cuestionario en el que se preguntaba por el número de horas semanales dedicadas a la enseñanza de la música en esta etapa educativa, el tipo de docente que la imparte o el año en que esta disciplina fue incluida, como materia obligatoria, en sus planes de estudios, entre otros asuntos. Los datos obtenidos ${ }^{3}$ nos mostraron una falta de homogeneidad inter-países que está dificultando el cumplimiento de varios objetivos marcados por la UE en políticas educativas.

La codificación de los documentos seleccionados nos ha permitido seleccionar los once informes que, desde nuestro punto de vista, mejor reflejan la postura de la UE sobre la importancia de la enseñanza de la música en las escuelas.

\section{Once documentos clave de la Unión Europea sobre educación musical ${ }^{4}$}

\subsection{Resolución del Parlamento Europeo de 10.02.1988 sobre la enseñanza y promoción de la música en la Comunidad Europea}

El Parlamento Europeo expone la importancia de la música en el desarrollo de la dimensión europea de la educación. Habla de la necesidad de fomentar el acceso a la vida musical y la participación activa en ella de un público cada vez más numeroso y joven, por lo que cree imprescindible intensificar la Educación Musical, sobre todo en los centros escolares partiendo, asimismo, del principio de que la enseñanza de esta materia es un derecho del ciudadano europeo. Por último, el Parlamento Europeo recomienda a la Comisión Europea y a los estados miembros que adopten medidas legislativas para intensificar la presencia de la música y la cultura musical en la formación y en la vida de todos los ciudadanos de la Comunidad y considera que a la música se le debe reservar un lugar adecuado en los programas de intercambios de estudiantes y profesores entre países de la Comunidad Europea.

\subsection{Conclusiones del Consejo de 21 de junio de 1994 sobre los aspectos artísticos y culturales de la educación (DO C 229 de 18.08.1994)}

El Consejo recuerda que el Tratado de la Unión Europea pone especial énfasis en la consecución de una educación de calidad. La introducción en los temas culturales y la iniciación en la creación y la interpretación artísticas pueden contribuir, en gran medida, al desarrollo armonioso de los individuos en la sociedad, por lo que dicha educación de calidad necesita apostar de manera decidida por una mayor aportación cultural y artística. Por último, el Consejo insta a la Comisión a que conceda a los aspectos culturales y artísticos de la educación la importancia que merecen.

Estos datos se pueden consultar en López García y De Moya (2015).

4 Todos los documentos a texto completo en http://eur-lex.europa.eu/homepage.html 


\subsection{Conclusiones del Consejo de 18 de diciembre de 1997 sobre el papel de la música en Europa (DO 98/C 1/04 de 03.01.1998)}

El Consejo invita a la Comisión a que presente propuestas que trabajen a favor de la mejora del acceso de un público más amplio a la música, con especial atención a la Educación Musical desde la edad más temprana, apoyando acciones innovadoras y ejemplares que pongan de relieve el cometido básico e integrador de la música en la sociedad.

5.4. Resolución del Parlamento Europeo sobre el papel de las escuelas y de la educación escolar en el fomento del acceso público a la cultura [(DO de 26.02.2004) - (2002/2268 (INI)]

El apartado 5 de la exposición de motivos se refiere a la Educación Artística y la creatividad. En uno de sus párrafos se expone que la educación cultural desde una edad temprana y la participación en actividades artísticas y culturales es un factor importante en el desarrollo de las capacidades y las destrezas para la vida. Además, la Educación Artística no debería tratar sólo la transmisión cultural, sino también el desarrollo del potencial creativo de los alumnos.

\subsection{Resolución del Parlamento Europeo, de 7 de junio de 2007, sobre el estatuto social de los artistas [2006/2249(INI)]}

Esta resolución considera que debe garantizarse de manera eficaz la integración de las enseñanzas artísticas en los programas académicos de los estados miembros, garantizando la formación artística y cultural del individuo desde la más temprana edad. Tras las consideraciones previas, se expone una serie de peticiones y recomendaciones divididas en seis temáticas distintas. La última de ellas, garantizar la formación artística y cultural desde la más temprana edad, compuesta por los apartados 34 a 39, define los términos y recomendaciones sobre la educación artística en los sistemas educativos nacionales.

5.6. Resolución del Parlamento Europeo, de 24 de marzo de 2009, sobre los estudios artísticos en la Unión Europea [2008/2226(INI)]

La exposición de motivos de la propuesta que la Comisión de Cultura y Educación presenta al Parlamento Europeo nos ofrece información relevante sobre la situación de la enseñanza artística en ese momento y, como consecuencia, de la necesidad de desarrollar y aprobar esta resolución en base a las siguientes afirmaciones:

- La enseñanza artística es considerada universalmente como un componente indispensable de la educación en su concepción clásica. En la actualidad, si bien es una asignatura obligatoria en muchos sistemas educativos de los 
distintos estados miembros, siguen existiendo importantes diferencias entre modelos de enseñanza artística en los mismos.

- Los programas de educación artística ayudan a las personas a descubrir la diversidad de las expresiones culturales, desarrollan su sensibilidad hacia éstas, y contribuyen a descubrir el placer por el arte y la cultura. Además, refuerzan las identidades y los valores individuales y colectivos, y protegen la diversidad cultural.

En las consideraciones iniciales de la resolución podemos leer lo siguiente (Resolución 2008/2226 [INI]):

- La educación artística y cultural... es un desafío fundamental que deben asumir los sistemas educativos de los estados miembros.

- La educación cultural y artística es un componente esencial en la formación de los niños y jóvenes, pues contribuye al desarrollo del libre albedrío, la sensibilidad y la apertura hacia los demás; que representa un gran desafío para la igualdad de oportunidades y es premisa para una verdadera democratización del acceso a la cultura.

- Es deplorable y se deplora que por imperativos económicos los estados miembros con demasiada frecuencia se vean impelidos a reducir el espacio destinado a las artes en sus políticas educativas generales.

- La educación artística... favorece unas relaciones más estrechas y fructíferas entre la educación, la cultura y las artes.

- Existen importantes disparidades entre los modelos de enseñanza artística vigentes en los distintos estados miembros.

- La educación artística debería ser elemento obligado en los programas educativos a todos los niveles escolares, a fin de favorecer la democratización del acceso a la cultura.

- La educación artística y cultural conlleva intrínsecamente una aspiración de educación ciudadana y participa en la configuración de las ideas y la realización personal en los planos intelectual, afectivo y corporal.

Partiendo de estas premisas, el Parlamento Europeo pide al Consejo, a la Comisión y a los estados miembros que:

- Definan el papel de la educación artística como herramienta pedagógica esencial para revalorizar la cultura en un mundo globalizado y multicultural.

- Establezcan estrategias comunes para la promoción de políticas de educación artística y de formación del profesorado especializado en esta disciplina.

- Animen a los representantes nacionales en el... Grupo de Trabajo de Educación y Cultura... a examinar el cometido de las artes en los diferentes contextos educativos (formal, informal y no formal) y en todos los niveles educativos... así como la formación necesaria para los profesores especializados.

Por último, recomienda el desarrollo común de un portal europeo para la formación artística y cultural y la inclusión de la educación artística en los currículos educativos de los estados miembros para asegurar el desarrollo y el fomento del modelo cultural europeo. 
5.7. Recomendación del Parlamento Europeo, de 2 de abril de 2009, sobre mejorar las escuelas: un programa de cooperación europea (2010/C 137 E/09)

La consideración "Q" de este documento resuelve que una educación general que comprenda materias tales como las artes y la música, puede contribuir al fomento de la realización personal, la confianza en uno mismo y el desarrollo de la creatividad y el pensamiento innovador. En este mismo sentido, la petición de mejora "19" considera que todos los niños deben disponer, desde las edades más tempranas, de oportunidades para adquirir competencias musicales, artísticas, manuales, físicas, sociales y cívicas y expone la creencia firme del Parlamento Europeo de que la Educación Musical, Artística y Física debe estar incluida de manera obligatoria en los planes de estudio de las escuelas.

\subsection{Conclusiones del Consejo, de 27 de noviembre de 2009, sobre la promoción de una generación creativa: desarrollar la creatividad y la capacidad innovadora de los niños y de los jóvenes a través de la expresión cultural y del acceso a la cultura (2009/C 301/08)}

En éstas, el Consejo señala que el acceso y la exposición a expresiones culturales, prácticas artísticas y obras de arte diversas desde una edad temprana son fundamentales para el desarrollo personal, la autoestima, la identidad y el sentido de pertenencia del individuo ya que, con ello, se dota a niños y jóvenes de competencias interculturales y de otras aptitudes importantes para la inclusión social y para la ciudadanía activa y las posibilidades de empleo futuro. Además, señala que la promoción de la cultura y de la expresión cultural en las escuelas y demás instituciones de enseñanza, así como en el entorno de la enseñanza no formal, ya sea a modo de asignaturas específicas como de otros planteamientos de aprendizaje que conecten campos del conocimiento distintos, contribuye al desarrollo completo del individuo, a la motivación y a un mejor aprendizaje, así como al desarrollo de la creatividad y de las habilidades de innovación.

\subsection{Educación artística y cultural en el contexto escolar en Europa (Eurydice,} 2010)

Este informe presenta un completo análisis sobre el estado actual de la Educación Artística, y de la Educación Musical como parte de esta área de conocimiento, tanto a nivel nacional como desde parámetros comparativos entre estados. En él quedan recogidos aspectos de gran interés como los diferentes modelos de Educación Artística, el tipo de docentes que imparten las materias que configuran esta disciplina, los modelos de organización curricular y de evaluación, el número de horas lectivas dedicadas a las enseñanzas artísticas y la formación del profesorado de materias artísticas en la educación general obligatoria, entre otros asuntos. 


\subsection{European Agenda for Culture. Open Method of Coordination. Working Group on developing synergies with education, especially arts education. Final Report. 2010}

En este documento se exponen y analizan dieciséis recomendaciones básicas, divididas en cuatro grandes bloques temáticos, para el desarrollo de sinergias en educación artística. Cada recomendación propone diferentes acciones que deberán ser favorecidas, en unos casos, y fortalecidas, en otros, tanto por los estados miembros como por la propia Unión Europea.

Tabla 2: Recomendaciones del grupo de trabajo a la Comisión y a los estados miembros

\begin{tabular}{|l|}
\hline Bloque I. Contenidos de la Educación Artística y Cultural \\
\hline Recomendación 1. Promover la interdisciplinariedad \\
\hline $\begin{array}{l}\text { Recomendación 2. Promover y reforzar el conocimiento y valoración del patrimonio des- } \\
\text { de un enfoque global }\end{array}$ \\
\hline
\end{tabular}

\section{Bloque II. Educación en TIC}

Recomendación 3. Estudiar y promover en cada Estado miembro nuevos servicios y estrategias para garantizar el acceso y la plena inclusión de las TIC en los ámbitos educativos y culturales

Recomendación 4. Implementar y mejorar las iniciativas de educación en TIC de los estados miembros, en particular las iniciativas que desarrollen la creatividad mediante las TIC, incluyendo procesos de evaluación con los que poder valorar los programas llevados a cabo y los resultados obtenidos

Recomendación 5. Reforzar las políticas de apoyo sobre la utilización de las TIC para el desarrollo de la creatividad

Recomendación 6. Valorar y promover el talento creativo y las habilidades creativas adquiridas por los niños y adolescentes

Recomendación 7. Involucrar a todas las instituciones educativas y culturales en el proceso de introducción y desarrollo de la creatividad mediante las TIC en los ámbitos educativos

Bloque III. El papel de las instituciones y las asociaciones culturales

Recomendación 8. Favorecer la cooperación entre las escuelas y las organizaciones culturales mediante políticas y estrategias a largo plazo, definiendo el papel de ambas instituciones y supervisando periódicamente los procesos realizados. De esta manera, la cooperación será más efectiva y sostenible y no quedará únicamente plasmada en un proyecto irrealizable.

Recomendación 9. Apoyar el intercambio de información, conocimientos y resultados entre los estados miembros sobre las políticas de cooperación entre las instituciones educativas y culturales, facilitando a las redes de información de la Unión Europea la realización de documentos e informes que puedan ser consultados desde los portales de educación y cultura de los que dispone la propia Unión 
Recomendación 10. Promover la cooperación entre las instituciones educativas y culturales interpaíses

Recomendación 11. Destinar partidas económicas a estas organizaciones para la creación de equipos de mediación y seguimiento que aseguren el cumplimiento de los proyectos puestos en marcha

Recomendación 12. Ofrecer el apoyo suficiente para la formación de profesores, artistas y otros profesionales de la cultura y asegurar los procesos de formación continua y permanente para estos

Recomendación 13. Realzar el papel de la educación artística y los efectos beneficiosos que ejercen sobre las personas en la sociedad actual

\section{Bloque IV. Evaluación}

Recomendación 14. Incluir programas de evaluación de las políticas nacionales desarrolladas

Recomendación 15. Contribuir en el desarrollo de programas de formación del profesorado y de los profesionales de la cultura en técnicas de evaluación, ofreciéndoles las herramientas necesarias para llevar a cabo dichos procesos evaluativos

Recomendación 16. Establecer un observatorio a nivel europeo. Éste deberá tener la capacidad de recoger los informes producidos por los estados miembros y de producir estudios analíticos y comparativos que sirvan de soporte para la mejora de la información sobre el estado de la educación artística y cultural de la Unión Europea

En cuanto a cómo se podría seguir trabajando en este campo, el grupo de trabajo propone la creación de equipos transnacionales dentro del formato MOC (Método Abierto de Coordinación), con el objetivo de identificar las sinergias existentes entre los diferentes estados miembros y, de esta manera, definir proyectos concretos. Estos equipos deberán contribuir a que tanto la Comisión como los estados miembros concreten su labor siguiendo el plan de acción propuesto en las recomendaciones anteriores.

\subsection{Conclusiones del Consejo, de 27 de mayo de 2015, sobre el papel de la educación infantil y primaria en el fomento de la creatividad, la innovación y la competencia digital (2015/C 172/05)}

Este informe pone en valor la necesidad de fomentar la creatividad, la innovación y la competencia digital a una edad temprana, y se exponen los beneficios potenciales para los jóvenes del fomento de estos tres ámbitos en la vida adulta.

Tras la presentación de los acuerdos adoptados, el Consejo insta a los estados miembros, teniendo presentes la subsidiariedad y la autonomía institucional, a que alienten a los proveedores de programas de formación inicial y continua, tanto para los futuros docentes como para los maestros en activo, a tener en cuenta métodos eficaces para fomentar la curiosidad, la experimentación, el pensamiento creativo y crítico y la comprensión cultural, siendo el arte, la música y el teatro disciplinas fundamentales para ello. 


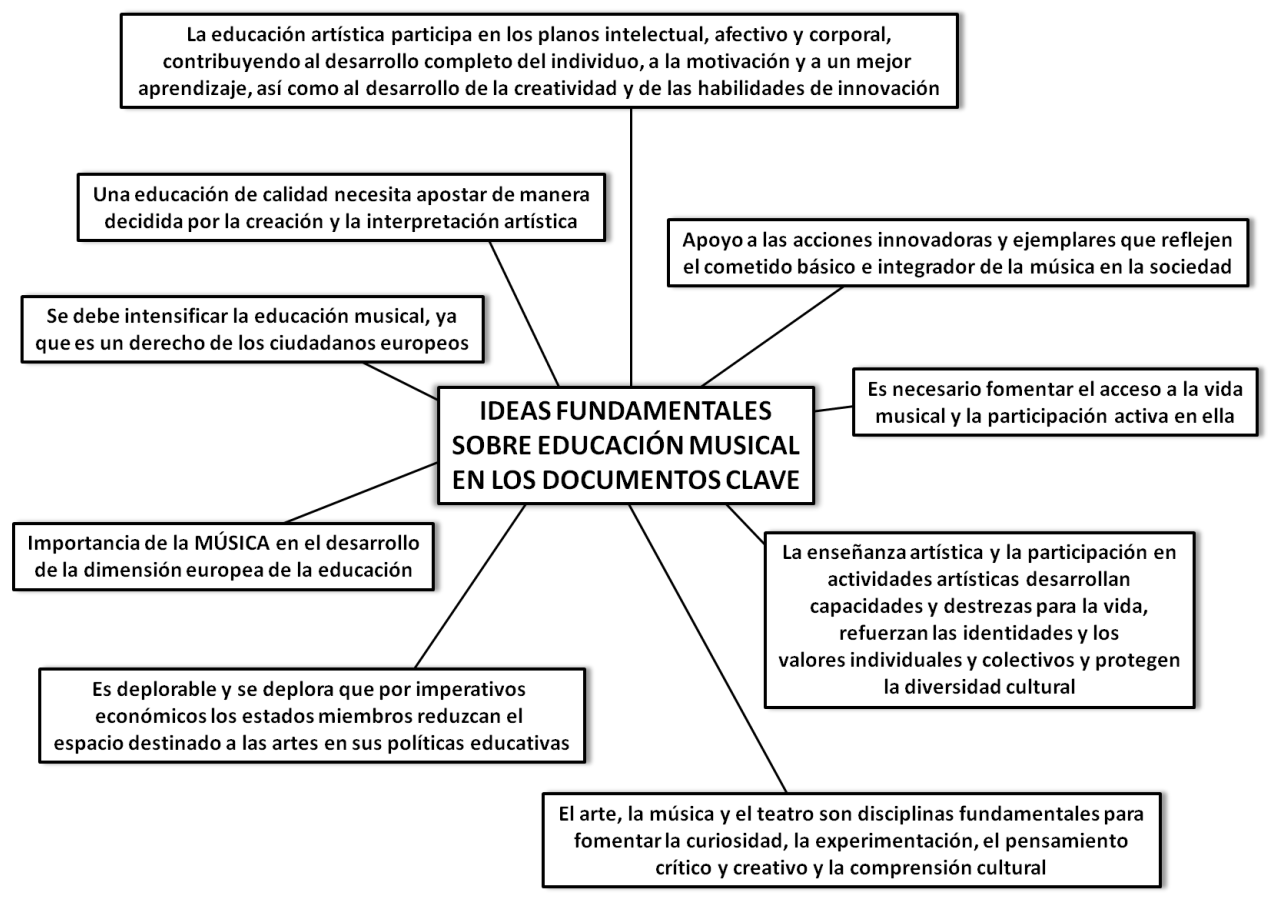

Figura 1: Ideas fundamentales recogidas en los documentos clave de la UE sobre Educación Artística y Educación Musical

Con respecto a la repercusión de estas indicaciones en los sistemas educativos nacionales, los cambios legislativos en materia educativo-musical derivados de la adaptación de los mismos al Espacio Europeo de Educación Superior (EEES) reflejan que los ministerios de educación están pasando por alto las recomendaciones ofrecidas por los diferentes organismos comunitarios en relación a esta área de conocimiento.

Centrándonos en los datos aportados por "Autores" (2015), podemos observar grandes diferencias en la configuración de esta materia en los planes de estudios nacionales destacando, entre otros aspectos, los distintos perfiles del profesorado encargado de su enseñanza, la carga lectiva, la configuración de la Educación Musical como una asignatura independiente o perteneciente al grupo de disciplinas del Área de Educación Artística o el año de inclusión de la misma en los currículos oficiales como materia obligatoria, entre otros asuntos.

Por otro lado, encontramos cierta conexión en los programas nacionales, que se estructuran alrededor de grandes bloques: escucha activa, movimiento y danza, música y TIC, interpretación, composición y creación musical, la voz y el canto o la lecto-escritura musical.

En relación a la evaluación, los veintiocho estados miembros mantienen un sistema de evaluación interna en la que el docente encargado de impartir la enseñanza de la música es quien valora y califica al alumnado. Además, nos llama la atención que solo cinco países (Estonia, Finlandia, Francia, Hungría y Reino Unido [Gales e Irlanda del Norte]) tienen datos nacionales relativamente recientes sobre la calidad de la enseñanza de las materias artísticas en sus sistemas educativos. 
Por el contrario, sirva como ejemplo el caso de Suiza que, en el año 2012, y por iniciativa popular, decidió incorporar a su Constitución una normativa que garantizara una educación musical de alta calidad en niños y jóvenes dentro de su sistema de educación obligatoria. Además, esta medida reivindica la figura del docente especialista y facilita el acceso de alumnos que destaquen en este ámbito a las escuelas de música y conservatorios. Este proceso, que culmina en 2016, tiene por objeto garantizar la presencia de la música y el canto en la formación obligatoria en todos los centros escolares del país, definir las competencias musicales que deberán adquirir los alumnos y la forma de evaluarlas y, finalmente, la organización y dotación del sistema educativo de cada cantón suizo de profesorado especializado y de instalaciones adecuadas.

El proceso está avalado por el Gobierno Central suizo que se compromete a velar por el cumplimiento de la normativa y a intervenir en aquellos cantones que incumplan con las directrices fijadas, sea cual sea la causa, para solucionar cualquier desigualdad entre regiones.

Esta situación ha despertado, de nuevo, el interés de la Unión Europea por conocer la realidad de las enseñanzas artísticas en las escuelas primarias de los países que la configuran. Fruto de ello, en diciembre de 2015 se publicaba el Handbook on cultural awareness and expression, en el que se recogen las reflexiones del grupo de expertos de la Unión sobre la Conciencia Cultural y la Expresión y se definen las implicaciones de estos nuevos términos en el desarrollo de las Competencias Clave, las buenas prácticas para los estados miembros y las recomendaciones del grupo.

Una vez terminado el curso escolar 2016-2017, dispondremos de los primeros datos para valorar cómo los ministerios de educación nacionales han analizado, gestionado y aplicado estas últimas recomendaciones.

\section{Conclusiones}

El análisis de los documentos seleccionados pone de manifiesto la importancia que la Unión Europea concede a la Educación Musical, y al resto de enseñanzas artísticas, en las edades tempranas. Sin embargo, las diferentes concepciones sobre política educativo-musical que mantienen los estados miembros, en virtud de sus competencias plenas en materia educativa, han generado una serie de disparidades inter-naciones que están ocasionando, no sólo desequilibrios a nivel curricular, sino serias dificultades a la hora de poner en marcha programas y proyectos comunitarios relacionados con la movilidad y el intercambio de estudiantes, de docentes y de artistas. Además, la inexistencia de criterios unificados está permitiendo cambios legislativos que parecen alejarse del camino marcado por las altas instituciones europeas, abriendo aún más, si cabe, la brecha de la desigualdad educativa que el denominado Proceso de Bolonia se había propuesto cerrar.

Con respecto a la correcta integración de las enseñanzas artísticas y musicales en los currículos de las etapas educativas obligatorias, si bien la preocupación de los organismos europeos queda patente en los textos analizados, echamos en falta la existencia de comisiones, ya sean estatales o comunitarias, encargadas de comprobar que los postulados de las altas instituciones de la Unión en esta materia se hagan efectivos en las legislaciones nacionales. En este sentido, "Autores" (2015) muestran 
cómo las directrices nacionales se desvían claramente de las comunitarias, lo que está generando malestar entre los profesionales de la educación musical, que ven con preocupación el futuro de la enseñanza de la música en los currículos oficiales.

No cabe duda de que aún queda mucho por hacer. La Unión Europea debe plantearse nuevos retos que le permitan controlar, en mayor medida, los cambios legislativos futuros en las políticas educativas nacionales. Y esto pasa, indefectiblemente, por establecer un currículo de mínimos supranacional que, sin menoscabar las singularidades nacionales, defina las líneas de actuación básicas en materia educativa; no parece tener mucho sentido recomendar, proponer, trabajar... en definitiva, abrir caminos cuyos recorridos no van más allá de las simples palabras.

Nos gustaría concluir con una reflexión de Jean Monnet que, analizando la importancia de las políticas educativas de la Unión Europea, no dudaba en afirmar que "si hubiera que rehacer todo desde el principio, habría que empezar por la cultura y la educación” (en Braga, 1995, p.206).

\section{Limitaciones y prospectivas}

La escasez de investigaciones similares a la nuestra ha dificultado nuestra labor, que se ha tenido que centrar en el análisis de documentos oficiales, de programaciones y currículos nacionales y de los datos ofrecidos por la red Eurydice y por los departamentos de Educación Primaria de los ministerios de educación de los veintiocho, por lo que ha sido sumamente complicado cotejar datos, ampliar bibliografía o realizar comparativas.

Con respecto a las prospectivas de futuro, este estudio supone un primer acercamiento con el que pretendemos que se abran nuevas vías de investigación y de reflexión sobre el grado de incidencia de las recomendaciones europeas, en materia de educación artística, en los sistemas educativos nacionales. Además, en el transcurso del estudio han surgido una serie de interrogantes que pueden marcar líneas de trabajo futuras relacionadas con la implicación de los estados miembros en el correcto desarrollo de las competencias artísticas, con la realización de informes comparativos inter-países actualizados, con los nuevos retos y responsabilidades de la Unión Europea en políticas educativas o con la creación de grupos de expertos nacionales encargados de controlar e informar sobre la incidencia de las recomendaciones de los diferentes organismos e instituciones de la UE con competencias en materia educativa en los sistemas educativos nacionales, entre otros asuntos. Todo ello, con la idea de crear un espacio educativo europeo, especialmente un espacio educativo-musical, coherente, unificado y equiparable facilitando, con ello, la movilidad de músicos, artistas, estudiantes y profesorado especialista, las oportunidades laborales y la homologación de títulos tal y como lo establecen las directrices comunitarias surgidas del denominado Proceso de Bolonia.

\section{Referencias bibliográficas}

Bardin, L. (2002). Análisis de contenido. Tres Cantos (Madrid): Akal.

Braga, P. (1995). Dimensión europea de la educación. Análisis de contenido de algunos documentos oficiales nacionales. Revista Española de Educación Comparada, 1, 205-217. 
Cheung, C. (2004). Retos de los responsables políticos para lograr una implantación eficaz: los cambios del currículo en la música. En ISME, Mundos sonoros por descubrir. Seminario de la Comisión ISME para la música en políticas culturales, educativas y en los medios de comunicación. XXVI Conferencia Internacional ISME, 11-16 de julio de 2014, Tenerife - España. Bilbao: ISME.

Comisión Europea (2011). Informe general sobre la actividad de la Unión Europea. 2011. Luxemburgo: Comisión Europea. Dirección General de Comunicación. Oficina de Publicaciones de la Unión Europea. Recuperado de http:/www10.iadb.org/intal/intalcdi/ PE/2012/09792.pdf, 15 de marzo de 2012.

De Baets, T. y Buchborn, T. (2014). European perspectives on music education 3. The reflective music teacher. Innsbruck: Helbling Languages.

Díaz Gómez, M. (2005). La educación musical en la escuela y el Espacio Europeo de Educación Superior. Revista Interuniversitaria de Formación del Profesorado, 19 (1), 23-37.

Diestro, A. (2009). De políticas, educación y ciudadanía europea. Pliegos de Yuste, 9-10, 55-66.

Dulzaides, M.E. y Molina, A.M. (2004). Análisis documental y de información: dos componentes de un mismo proceso. ACIMED, 2. Recuperado de http://eprints.rclis.org/5013/1/ analisis.pdf, 14 de octubre de 2014.

$\mathrm{EA} / \mathrm{mS}$ (2013). Music education in Europe.

Etxeberria, F., Ayerbe, P., Garagorri, X. y Vega, A. (2000). Políticas educativas en la Unión Europea. Barcelona: Ariel Practicum.

Eurydice (EACEA) (2010a). Educación artística y cultural en el contexto escolar en Europa. Madrid: Secretaría General Técnica. Subdirección General de Documentación y Publicaciones. Recuperado de http://eacea.ec.europa.eu/Education/eurydice/documents/ thematic_reports/113ES.pdf, 21 de marzo de 2012.

Eurydice (EACEA) (2010b). Structures of education and training systems in Europe. 2009/2010 Edition. Bruselas: Eurydice. Recuperado de http://biblioteka-krk.ibe.edu.pl/ opac_css/doc_num.php?explnum_id=293, 21 de marzo de 2012.

Eurydice (EACEA) (2010c). Organisation of the education system in... Bruselas: Eurydice. Recuperado de http://www.eures.ee/public/documents/0/Hariduss $\% \mathrm{C} 3 \% \mathrm{BC}$ steem $\% 20$ Saksamaal\%20inglise\%20keeles.pdf, 21 de marzo de 2012.

Eurydice (EACEA) (2011) . National system overview on education systems in Europe. 2011 Edition. Bruselas: Eurydice. Recuperado de http:/www.erisee.org/downloads/2013/2/r/ National\%20education\%20system\%20overview\%202011\%20ENg.pdf, 3 de abril de 2012.

Eurydice (EACEA) (2014). The structure of the European Education Systems 2014/2015: Schematic Diagrams. Bruselas: Eurydice - Facts and Figures. Recuperado de http:// eacea.ec.europa.eu/education/eurydice/documents/facts_and_figures/education_structures_EN.pdf, 7 de octubre de 2015.

Fontaine, P. (2011). Doce lecciones sobre Europa. Luxemburgo: Comisión Europea. Dirección General de Comunicación. Oficina de Publicaciones Oficiales de la Unión Europea. Recuperado de http://bookshop.europa.eu/es/doce-lecciones-sobre-europa-edici-n-de-2010-pbNA3110652/, 23 de febrero de 2014.

Fontaine, P. (2014). Doce lecciones sobre Europa. Comprender las políticas de la Unión

Las referencias bibliográficas de Eurydice 2010b, 2010c y 2011 son genéricas. Para realizar esta investigación se han revisado cada uno de los informes publicados correspondientes a los veintiocho Estados miembros de la Unión Europea. 
Europea. Bruselas: Comisión Europea. Dirección General de Comunicación. Publicaciones. Recuperado de http://bookshop.europa.eu/is-bin/INTERSHOP.enfinity/WFS/ EU-Bookshop-Site/es_ES/-/EUR/ViewPublication-Start?PublicationKey=NA0213714, 4 de noviembre de 2015.

Ibáñez - Martín, J.A. (2002). Tendencias en la política de la educación de la Unión Europea. Arbor, 681, 39-53.

IBE. UNESCO (2012) ${ }^{6}$. World data on education. VII Ed. 2010/2011. Ginebra: UNESCO. International Bureau of Education. Recuperado de http://www.unesco.org/new/es/education/resources/online-materials/publications/, 8 de abril de 2014.

Lauret, J.M. y Marie, F. (Eds.) (2010). European agenda for culture. Open method of coordination. Working group on developing synergies with education, especially arts education. Final report. Recuperado de file://C:/Users/Pepe\%20L\%C3\%B3pez/Downloads/ i007395\%20(1).pdf, 2 de marzo de 2013.

López García, N.J. y De Moya, M.V. (2015). La música en las escuelas europeas. Situación actual de la Educación Musical en los currículos de Primaria de los Estados miembros de la Unión Europea. Albacete: Liberlibro.com.

Paricio, M.S. (2005). Política educativa europea. Revista de Educación, 337, 251-278.

Stavrou, N.E. y Stakelum, M. (Eds.) (2015). European perspectives on music education 4. Every learner counts. Democracy and inclusión. Innsbruck: Helbling Languages.

Subirats, M.A. (2005). La educación musical en el espacio europeo de educación superior. Revista Interuniversitaria de Formación de Profesorado, 19 (1), 39-51.

Valle, J.M. (2004). La política educativa de la Unión Europea: fundamentos, evolución histórica y propuesta de un modelo para su análisis crítico. Revista Española de Educación Comparada, 10, 17-59.

Valle, J.M. (2006a). La Unión Europea y su política educativa. Tomo I. La integración europea. Madrid: Ministerio de Educación y Ciencia. Secretaría General de Educación. CIDE.

Valle, J.M. (2006b). La Unión Europea y su política educativa. Tomo II. Medio siglo de acciones en materia educativa. Madrid: Ministerio de Educación y Ciencia. Secretaría General de Educación. CIDE.

Zaldívar Gracia, A. (2005). Las enseñanzas musicales y el nuevo espacio europeo de educación superior: el reto de un marco organizativo adecuado y la necesidad de la investigación creativa y performativa. Revista Interuniversitaria de Formación del Profesorado, $19(1), 95-122$.

Para esta investigación se han recuperado los informes correspondientes a los veintiocho Estados miembros de la Unión Europea. 